\title{
Asociación entre conocimiento y prácticas sobre la infección por VIH en mujeres peruanas: análisis secundario de encuesta nacional
}

\author{
Association between knowledge and practices on HIV infection in Peruvian women: \\ secondary analysis of national survey
}

Christoper A. Alarcon-Ruiz1, Mariela Vargas², Paula Heredia², Manuel O. Huamán², Marlon Yovera-Aldana ${ }^{3}$ y Christian R. Mejia ${ }^{4}$

1Universidad Científica del Sur, Lima, Perú.
${ }^{2}$ Instituto de Investigación en Ciencias Biomédicas, Universidad Ricardo Palma, Lima, Perú.
${ }^{3}$ Escuela de Medicina, Universidad Nacional de Piura, Piura, Perú.
${ }^{4}$ Universidad Continental, Huancayo, Perú.

Fuentes de financiamiento: Autofinanciado.

Los investigadores declaran no tener conflicto de interés.

Recibido: 27 de marzo de 2020 (segunda versión: 15 de septiembre de 2020) / Aceptado: 2 de noviembre de 2020

\section{Resumen}

Introducción: Las mujeres en edad reproductiva podrían considerarse como una población vulnerable a contraer la infección por VIH. La relación entre conocimiento y prácticas no está descrita en un contexto donde los conocimientos, actitudes y prácticas sobre el VIH son inadecuados. Objetivo: Determinar la asociación entre conocimientos y prácticas sobre la infección por VIH en mujeres peruanas en edad reproductiva. Material y Métodos: Análisis de Encuesta Demográfica y de Salud Familiar 2019, cuya muestra fue probabilística, estratificada y bietápica. El análisis consideró a mujeres en edad reproductiva sexualmente activas. Se definió "conocimientos adecuados" a los puntajes superiores al tercil superior de las observaciones al responder 18 preguntas y se determinó la asociación con cada una de las cinco prácticas. Se utilizó estadística descriptiva e inferencial -bivariada y multivariada- con regresión de Poisson para análisis poblacionales. Resultados: La media de respuestas correctas fue 12,6 (IC95\%: 12,5-12,7). El 19,2\% había usado preservativo en su última relación sexual. Un nivel de conocimientos adecuado estuvo asociado al uso de preservativo en la última relación sexual (RPa: 1,20; IC95\%: 1,06-1,36), haber tenido una infección de transmisión sexual y con haberse realizado una prueba diagnóstica para infección por VIH. Conclusiones: Considerando una baja prevalencia de uso de preservativo en la última relación sexual de mujeres peruanas en edad reproductiva, los conocimientos generales adecuados sobre la infección por VIH se asocian con haber realizado esta práctica.

Palabras clave: conocimiento; mujeres; infección por VIH; Perú.

\section{Abstract}

Background: Reproductive-age women are a vulnerable population for HIV infection. The relationship between knowledge and practices is not well described in a context where levels of knowledge, attitudes, and practices about HIV infection are inadequate. Aim: To determine the association between knowledge and practices about HIV infection in Peruvian reproductive-age women. Methods: Analysis of secondary data from the 2019 Demographic and Family Health Survey. It got a probabilistic, stratified, and two-stage sample. We included reproductive-age and actively sexual women. Scores above the upper tertile of the observations were defined as "adequate knowledge" by answering 18 questions. Then, we determined the association between adequate knowledge with each of the five practices. Descriptive and inferential statistics -bivariate and multivariate- were used with the Poisson regression for survey analysis. Results: The mean of correct answers was 12.6 (95\% CI: 12.5 - 12.7). 19.2\% of Peruvian women had used a condom in the last sexual relationship. An adequate level of knowledge was associated with the use of condoms in the last sexual intercourse (PRa: 1.20; 95\% CI:1.06 - 1.36), with having a sexually transmitted infection in the last year, and with having a diagnostic test for HIV infection. Conclusions: Considering a low prevalence of condom use in the last sexual intercourse of Peruvian women of reproductive age, adequate general knowledge about HIV infection has associated with the accomplishment of this practice.

Keywords: knowledge, women, HIV infection, Peru. 


\section{Introducción}

Desde su aparición, más de 74,9 millones de personas se han infectado con el virus de inmunodeficiencia humana (VIH), causando la muerte de alrededor de 32 millones de personas ${ }^{1}$. Actualmente, los hombres que tienen sexo con otros hombres, los(as) trabajadores(as) sexuales, las personas trans-género, las personas que utilizan drogas inyectables, y los prisioneros, son grupos poblacionales claves en la lucha contra el $\mathrm{VIH}^{2}$. Sin embargo, en los últimos años, las mujeres se han comenzado a considerar como una de las poblaciones más vulnerables a contraer la infección por $\mathrm{VIH}^{3}$. Es así que, la incidencia de infección por VIH en mujeres jóvenes va en aumento, superando a sus pares masculinos ${ }^{4}$, reflejándose en cifras mundiales de 2,4 millones de adolescentes y mujeres jóvenes infectadas por $\mathrm{VIH}^{5}$. Particularmente, en este contexto, las mujeres son un grupo clave, pues se encuentran sometidas a la violencia de género y tienen un limitado acceso a la salud, las cuales están relacionadas con peores desenlaces en el contexto de la infección por $\mathrm{VIH}^{6-8}$.

El panorama en Latinoamérica es más importante aún, pues la violencia de género presente en la región es un problema de salud pública por enfrentar'. Es así que, la proporción de mujeres con infección por VIH ha aumentado desde 1994 hasta la actualidad, creciendo particularmente en Centroamérica y en poblaciones indígenas ${ }^{10}$. En el Perú, incluso la violencia de género es particularmente importante y alta ${ }^{11}$, pues durante el año 2018, más de $25 \%$ de los nuevos casos de infección por VIH notificados son mujeres y se calcula que la probable edad de exposición al virus es de 21 años ${ }^{12}$.

Esta pandemia ha obligado que la mayoría de los países implementen diferentes estrategias de intervención, con el objetivo de disminuir la transmisión del $\mathrm{VIH}^{13}$. Las intervenciones que buscan aumentar el conocimiento sobre la infección por VIH y su transmisión, harían a las personas capaces de identificar factores de riesgo y favorecería la modificación de conductas sexuales de riesgo ${ }^{14,15}$. Algunas prácticas sexuales de riesgo han estado asociadas a la infección por VIH y otras infecciones de transmisión sexual (ITS) ${ }^{16,17}$, sobre todo en países en vías de desarrollo ${ }^{18-20}$. Es así que, múltiples intervenciones han sido enfocadas hacia el cambio de estas prácticas, para la mejora de las habilidades, el conocimiento y la capacidad para protegerse del $\mathrm{VIH}^{21}$. Sin embargo, no siempre un adecuado conocimiento se relacionaría con no realizar prácticas sexuales de riesgo, especialmente en poblaciones vulnerables ${ }^{22,23}$. En el Perú, los conocimientos sobre la infección por VIH en mujeres no es adecuado, y su relación con algunas prácticas relacionadas al VIH no ha sido estudiado en grandes poblaciones ${ }^{24}$. Sólo se tienen reportes de proyectos nacionales, destinados a la prevención en salud sexual y reproductiva, sobre todo en comunidades nativas ${ }^{25,26}$. Por lo que, el objetivo del estudio fue determinar la asociación entre conocimientos y prácticas sobre la infección por VIH en mujeres peruanas en edad reproductiva.

\section{Material y Método}

Diseño y población. Se realizó un análisis transversal y analítico de datos secundarios, de la Encuesta Demográfica y de Salud Familiar (ENDES), que se ejecutó en el Perú el año 2019. Esta encuesta se realizó sobre una muestra probabilística de tipo equilibrado, bietápica, estratificada e independiente, por cada región, por área urbana y rural. Esta muestra es representativa del país para el año 2019, urbano/rural, y para cada región geográfica (Lima Metropolitana, Costa, Sierra, Selva). La unidad de muestreo primaria es la vivienda particular, y la unidad de investigación son los residentes habituales de los hogares elegibles, que hayan pernoctado la noche anterior a la encuesta. La encuesta tiene tres cuestionarios: Hogar, individual y de salud ${ }^{27}$. En el presente análisis se usó el cuestionario individual, que tuvo como población objetivo a las mujeres en edad reproductiva (MER) de 12 a 49 años, de cada vivienda elegida.

Se usó las bases de datos de los módulos 72 (conocimientos de VIH y uso de condón), 66 (datos básicos de MER) y 71 (nupcialidad). Se incluyó a las MER (12-49 años) que respondieron a la encuesta ENDES 2019, en su módulo $72(\mathrm{n}=33.311)$. Se excluyó aquellas que nunca habían tenido relaciones sexuales (Pregunta V525, “Cuántos años tenía Ud. cuando tuvo su primera relación sexual"?), a las mujeres embarazadas (Pregunta V623) y las que no tenían información de cualquiera de las cinco preguntas que evalúan prácticas relacionadas al VIH o cualquiera de las 18 preguntas que evalúan los conocimientos sobre la infección por VIH.

\section{Variables}

Las variables desenlace fueron cada una de las cinco prácticas relacionadas con la infección por VIH realizadas por las MER encuestadas, que se encontraban disponibles en el ENDES 2019. La variable "Tener más de dos parejas sexuales en el último año" (Sí/No) se creó al dicotomizar la pregunta “¿En total, con cuántos hombres diferentes ha tenido Ud. relaciones sexuales en los últimos 12 meses?". Las respuestas de 0 a 2 se categorizó como "No", y a las respuestas de más de 2 como "Sí". Para la variable "Usar preservativo en la última relación sexual" (Sí/No) se usó la pregunta "¿La última vez que Ud. tuvo relaciones sexuales con su última pareja sexual, usaron condón?". Para la variable "Tener diagnóstico de una ITS en el último año" (Sí/No) se usó la pregunta "En los últimos 12 meses, ¿Le han diagnosticado a Ud. alguna enfermedad de transmisión sexual?”. Para la variable “Consumir bebida 
alcohólica en última relación sexual" se usó la pregunta "La última vez que tuvieron relaciones sexuales ¿alguno de los dos tomó alguna bebida alcohólica?". Finalmente, para la variable "Haberse realizado alguna vez prueba diagnóstica para VIH” se usó la pregunta “¿Alguna vez se ha hecho la prueba para saber si tiene el virus que causa el SIDA?". En caso que alguna de estas preguntas tenga respuestas Sí, No, y No sabe/No responde, se consideró la respuesta "No sabe/No responde" como "No".

Para medir el nivel de los conocimientos sobre la infección por VIH se consideró 18 preguntas que se tenían disponibles en la encuesta ENDES 2019. Estas preguntas evaluaban conocimientos sobre factores de riesgo, transmisión (sexual, materna y no sexual), prevención (uso de preservativo) y diagnóstico de la infección por VIH. Se consideró la respuesta correcta para cada pregunta y, en caso de responder a alguna pregunta con "No sé/No responde", se consideró como una respuesta incorrecta. Luego, se calculó la sumatoria de respuestas correctas para cada encuestada. Se consideró que una MER tenía un nivel de conocimientos adecuado si se encontraba en el tercil superior del número total de respuestas correctas, en comparación con las que se encontraban en los dos terciles inferiores (nivel de conocimiento no adecuado).

Además, se incluyó las variables: edad en años, estado civil (soltera, casada, conviviente, divorciada, separada y viuda), región (Lima Metropolitana, Resto de Costa, Sierra, y Selva), tipo de residencia (urbana y rural), máximo nivel educativo aprobado (ninguno, educación primaria, secundaria, técnico, universitario, y post grado) e índice de riqueza (muy pobre, pobre, medio, rico, muy rico).

\section{Procedimientos}

Se descargó los módulos de datos directamente del portal del INEI, importados y unidos con el software estadístico Stata v16 (STATA Corporation, College Station, Texas, USA). Se usó como referencia la base de datos del módulo 72 (conocimientos de VIH y uso de condón) (RE758081). Luego, se unió con las bases de datos del módulo 66 (datos básicos de MER) (REC0111 y REC91), tomando a la variable "CASEID" como la variable de unión entre las bases. Se excluyó las observaciones de las bases REC0111 y REC91 que no lograron encontrar un par con la base principal RE758081. Finalmente, se unió con la base de datos del módulo 71 (nupcialidad) (RE516171), de la misma manera mencionada previamente. Antes del análisis estadístico se crearon las variables a analizar y se excluyeron las observaciones según los criterios de selección.

\section{Análisis estadístico}

Se realizó todos los análisis siguiendo las especificaciones del muestreo complejo del ENDES 201927,28. Esto se hizo mediante la especificación de los estratos, pesos y la unidad primaria de muestreo con el comando svyset en el software estadístico Stata v16 (STATA Corporation, College Station, Texas, USA). Se describió la prevalencia y sus intervalos de confianza al 95\% (IC95\%) de las variables categóricas dependientes e independientes. Asimismo, se reportó las medias e IC95\% para las variables edad y puntaje de conocimientos. Además, se realizó análisis bivariado para comparar la prevalencia de un conocimiento adecuado con cada una de las prácticas relacionadas con VIH, con la prueba de $\chi^{2}$. Para evaluar el tamaño de efecto entre las prácticas relacionadas con la infección por VIH (variable dependiente) y los conocimientos adecuados (variable explicativa), se usó un modelo de regresión de Poisson, con función de enlace log, para calcular las razones de prevalencia ajustadas ( $\mathrm{RPa}$ ), con sus respectivos IC de 95\%. En los modelos ajustados, se consideró como variables que confunden, al estado civil, región natural de vivienda, tipo de residencia, nivel de educación, nivel de pobreza y edad. Se realizó estos modelos con la regresión SVY para análisis poblacionales. Se realizó cinco modelos separados para cada variable dependiente, utilizando la categoría de conocimiento no adecuado, como referencia. Se consideró significativo a los valores $\mathrm{p}$ menores de 0,05 .

\section{Ética}

La base de datos, los glosarios de términos y los materiales usados son de dominio público por el Instituto Nacional de Estadística e Informática (http://iinei.inei. gob.pe/microdatos/).

\section{Resultados}

La base de datos de conocimientos de la infección por VIH y uso de condón encuestó a 33.311 MER, las que se mantuvieron luego de combinarla con las demás bases de datos. Se excluyó 9.076 (27,2\%) registros, porque no cumplían con los criterios de selección, obteniendo finalmente 24.235 observaciones en la muestra final para el análisis (Figura 1). El rango de edad fue de 14 a 49 años, con una media de 33,3 años (IC95\%: 33,1 - 33,5 años). Las MER eran en su mayoría convivientes (47,5\%), vivían en Lima Metropolitana (40,3\%), residían en una zona urbana $(82,7 \%)$, con educación secundaria completa $(41,1 \%)$ (Tabla 1$)$.

Todas las mujeres encuestadas respondieron correctamente a las preguntas sobre el haber oído del VIH y el conocer un lugar donde se pueda realizar la prueba para detección del virus que causa el síndrome de inmunodeficiencia humana (SIDA). Por el contrario, la menor proporción de respuestas correctas eran las que preguntaban sobre la transmisión del VIH de madre al niño durante el parto $(19,3 \%)$ y la lactancia $(29,1 \%)$ 


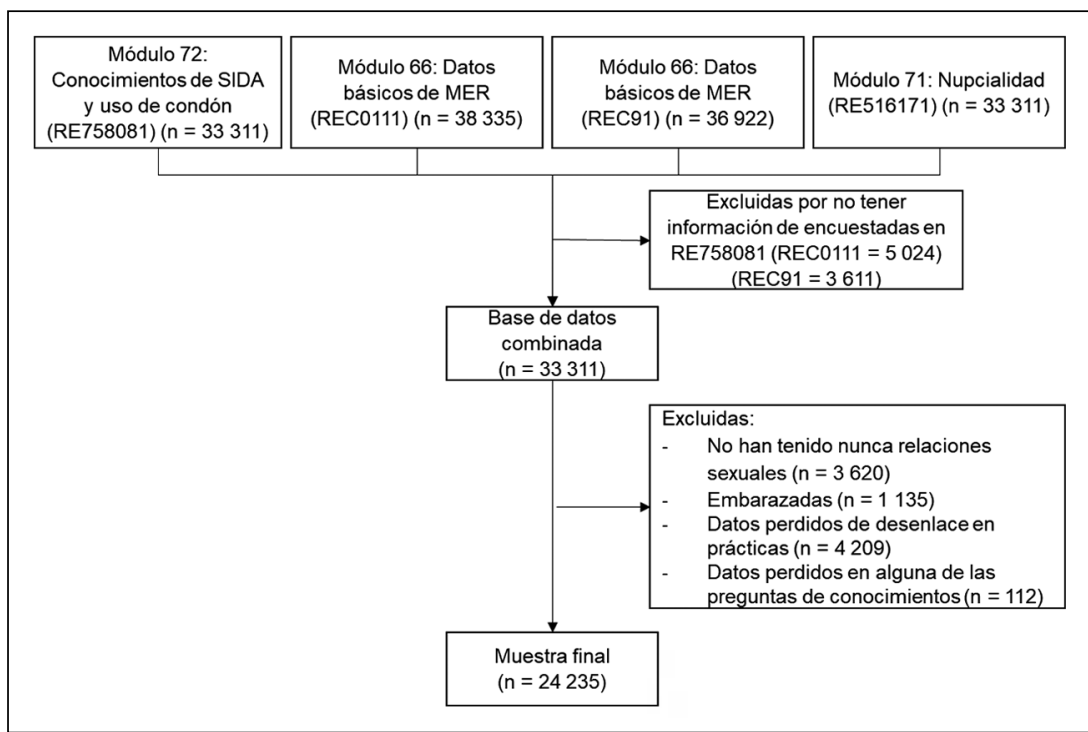

Figura 1. Flujograma de selección de participantes incluidas en el análisis, ENDES 2019. MER Mujeres en edad reproductiva, SIDA: síndrome de inmunodeficiencia adquirida.

(Tabla 2). Con respecto al conocimiento global de las MER, se obtuvo una media de 12,6 respuestas correctas (IC95\%: 12,5-12,7) con un rango de 2 a 18 respuestas correctas. La mayoría de las MER encuestadas tuvo entre 12 y 15 preguntas correctas $(52,6 \%)$, y $26,7 \%$ tenía un conocimiento adecuado. Con respecto a las prácticas, 19,2\% (IC95\%: 18,2-20,3) de las MER peruanas había usado preservativo en su última relación sexual. Por otro lado, $35,2 \%$ de las MER no casadas ni convivientes había usado preservativo en su última relación sexual. El 4,8\% (IC95\%: 4,2-5,5) de las MER peruanas había tomado una bebida alcohólica en su última relación sexual, $0,8 \%$ (IC95\%: 0,6-1,1) había tenido una ITS en el último año, $0,3 \%$ (IC95\%: 0,2-0,5) había tenido más de dos parejas sexuales en el último año y 79,6\% (IC95\%: 78,5-80,6) se había realizado alguna vez una prueba diagnóstica para la infección por VIH.

La prevalencia de prácticas relacionadas al VIH variaba según el nivel de conocimiento sobre la infección por VIH de las MER. Se encontró diferencias estadísticamente significativas de un conocimiento adecuado en las MER que habían usado preservativo $(\mathrm{p}<0,001)$ y que habían consumido alguna bebida alcohólica en su última relación sexual $(\mathrm{p}=0,029)$. Además, un buen conocimiento se asoció también con haber tenido un diagnóstico de una ITS en el último año $(p=0,018)$, y con haberse realizado alguna vez una prueba diagnóstica para la infección por VIH $(\mathrm{p}<0,001)$ (Tabla 3$)$.

La prevalencia de uso de preservativo fue $20 \%$ más (RPa: 1,20; IC95\%: 1,06-1,36; valor $p=0,003$ ) en las MER con nivel de conocimiento adecuado, en comparación con las que tenían un conocimiento no adecuado. De
Tabla 1. Características de las mujeres peruanas en edad reproductiva sexualmente activas incluidas en el estudio (n: 24.235), ENDES 2019

$\begin{array}{lrc}\text { Variable } & \mathbf{n}(\mathbf{\%}) & \mathbf{I C 9 5 \%} \\ \text { Estado civil } & & \\ \text { Conviviente } & 13.725(47,5) & 46,2-48,8 \\ \text { Casada } & 6.073(26,5) & 25,3-27,7 \\ \text { Soltera } & 2.361(15,8) & 14,7-16,9 \\ \text { Separada } & 2.018(9,7) & 8,9-10,5 \\ \text { Divorciada } & 39(0,4) & 0,2-0,7 \\ \text { Viuda } & 19(0,1) & 0,1-0,1 \\ & & \\ \text { Región } & & \\ \text { Lima Metropolitana } & 3.291(40,3) & 38,9-41,8 \\ \text { Resto de la Costa } & 7.416(24,0) & 23,0-25,0 \\ \text { Sierra } & 7.474(21,9) & 20,9-23,0 \\ \text { Selva } & 6.054(13,7) & 12,9-14,6 \\ & & \\ \text { Residencia } & & \\ \quad \text { Urbana } & & \\ \text { Rural } & 17.623(82,7) & 82,0-83,4 \\ & 6.612(17,3) & 16,6-18,0 \\ \text { Nivel educativo máximo } & & \\ \quad \text { Ninguno } & 357(1,2) & 1,0-1,4 \\ \text { Primaria } & 4.536(15,8) & 15,0-16,7 \\ \text { Secundaria } & 10.816(41,1) & 39,8-42,4 \\ \text { Técnico } & 4.561(20,3) & 19,2-21,4 \\ \text { Universitario } & 3.661(19,6) & 18,4-20,8 \\ \text { Post grado } & 304(2,1) & 1,7-2,7\end{array}$

Índice de pobreza

$\begin{array}{lll}\text { Muy pobre } & 5.909(15,6) & 14,9-16,3 \\ \text { Pobre } & 6.493(21,0) & 19,9-22,0 \\ \text { Medio } & 5.040(21,4) & 20,3-22,6 \\ \text { Rico } & 3.932(21,5) & 20,2-22,9 \\ \text { Muy rico } & 2.861(20,6) & 19,2-22,0\end{array}$

Edad (años)* $\quad 33,3(33,1-33,5)$

IC95\%: Intervalo de confianza al 95\%. *Media e IC95\%

la misma manera, la prevalencia de tener un diagnóstico de una ITS en el último año fue $122 \%$ mayor (RPa: 2,22; IC95\%: $1,14-4,37$; valor $\mathrm{p}=0,020$ ) y la prevalencia de haberse realizado alguna vez una prueba diagnóstica para la infección por VIH fue $11 \%$ mayor (RPa: 1,11; IC95\%: $1,08-1,14$; valor $\mathrm{p}<0,001)$ en las MER con nivel de conocimiento adecuado. Estos estimados estuvieron ajustados por el estado civil, la región natural donde vivían, el tipo de residencia, el nivel de educación, el nivel de pobreza y la edad de las encuestadas. El resto de las prácticas no se encontraban asociadas con un nivel de conocimiento adecuado (Tabla 3 ). 
Tabla 2. Conocimientos sobre VIH en mujeres peruanas en edad reproductiva sexualmente activas (n: 24.235), ENDES 2019

Conocimientos de VIH

¿Ud. ha oído hablar de la infección por VIH?

¿Conoce usted algún lugar donde una persona pueda hacerse la prueba para saber si tiene el virus que causa el SIDA (VIH)?

¿Cree Ud. que una persona puede hacer algo para prevenir el virus que causa el SIDA (VIH)?

¿Cree Ud. que las personas que adquirieron el virus que causa el SIDA (VIH) fue por castigo divino?

¿Cree Ud. que las personas tienen menos riesgo de adquirir el virus que causa el SIDA (VIH), si tienen una sola pareja sexual que no esté infectado y que no tenga otras parejas?

¿Es posible que una persona que parece saludable esté infectada con el virus que causa el SIDA (VIH)?

¿Cree Ud. que las personas que usan condón tienen menos riesgo de adquirir el virus que causa el SIDA (VIH)?

¿Cree Ud. que las personas pueden adquirir el virus que causa el SIDA (VIH) por dar un abrazo, beso o caricias a una persona infectada?

¿Conoce un lugar donde podría conseguir un condón?

(Aparte del SIDA), ¿Ud. ha oído hablar de (otras) infecciones que se pueden transmitir a través de las relaciones sexuales?

¿Cree Ud. que las personas que no tienen relaciones sexuales tienen menos riesgo de adquirir el virus que causa el SIDA (VIH)?

¿Cree Ud. que las personas pueden adquirir el virus que causa el SIDA (VIH) compartiendo utensilios como cubiertos, vasos, etc. con una persona infectada?

¿Cree Ud. que las personas pueden adquirir el virus que causa el SIDA (VIH) por compartir baños, duchas y/o piscinas?

¿EI virus que causa el SIDA $(\mathrm{VIH})$, puede ser transmitido de una madre infectada al niño durante el embarazo?

¿Cree Ud. que las personas pueden adquirir el virus que causa el SIDA $(\mathrm{VIH})$ por la picadura de un mosquito?

¿Conoce dos o más ITS aparte del SIDA $(\mathrm{VIH})$ ?

¿EI virus que causa el SIDA (VIH), puede ser transmitido de una madre infectada al niño durante la lactancia?

¿El virus que causa el SIDA (VIH), puede ser transmitido de una madre infectada al niño durante el parto?

Respuesta
correcta
n (\%)

$24.235(100,00)$

$24.235(100,00)$

$22.176(93,0)$

$92,4-93,5$

$20.582(87,5) \quad 86,7-88,2$

$21.013(87,3) \quad 86,5-88,1$

$195.563(82,8) \quad 81,9-83,7$

$19.834(82,6) \quad 81,6-83,6$

$18.442(80,7) \quad 79,8-81,6$

$18.253(78,2) \quad 77,2-79,2$

$17.749(78,2) \quad 77,2-79,2$

$18.304(73,8) \quad 72,6-74,9$

$15.730(68,1) \quad 66,9-69,2$

$14.947(66,1) \quad 64,9-67,3$

$13.463(58,1) \quad 56,8-59,4$

$9.108(39,6) \quad 38,3-40,9$

$7.821 \quad(35,7) \quad 34,4-37,1$

$7.602(29,1) \quad 27,9-30,4$

$4.361 \quad(19,3) \quad 18,3-20,4$

IC95\%: Intervalo de confianza al 95\%.

Tabla 3. Asociación entre un nivel de conocimiento adecuado y las prácticas de VIH en mujeres peruanas en edad reproductiva sexualmente activas, ENDES 2019

\begin{tabular}{|c|c|c|c|c|c|c|c|}
\hline \multirow[t]{2}{*}{ Prácticas } & \multicolumn{2}{|c|}{$\begin{array}{l}\text { Conocimiento adecuado; } \\
\text { n (\%)* }\end{array}$} & \multirow[t]{2}{*}{ Valor $p^{* *}$} & \multicolumn{2}{|c|}{ Bivariado } & \multicolumn{2}{|c|}{ Multivariado*** } \\
\hline & No & Sí & & RPc & IC95\% & $\mathbf{R P a}$ & IC95\% \\
\hline Usar preservativo en la última relación sexual & $2652(14,4)$ & $1225(21,1)$ & $<0,001$ & $1,48^{f}$ & $1,31-1,67$ & $1,20^{\ddagger}$ & $1,06-1,36$ \\
\hline Consumir bebida alcohólica en la última relación sexual & $638(3,5)$ & $261(4,5)$ & 0,029 & $1,34^{\ddagger}$ & $1,03-1,74$ & 1,07 & $0,79-1,44$ \\
\hline Tener más de dos parejas sexuales en el último año & $19(0,1)$ & $13(0,2)$ & 0,369 & 1,72 & $0,52-5,68$ & & \\
\hline Tener diagnóstico de una ITS en el último año & $106(0,6)$ & $53(0,9)$ & 0,018 & $2,21^{\ddagger}$ & $1,13-4,31$ & $2,22^{\ddagger}$ & $1,14-4,37$ \\
\hline Haberse realizado alguna vez una prueba diagnóstica para VIH & $15312(83,0)$ & $5413(93,7)$ & $<0,001$ & $1,14^{f}$ & $1,11-1,18$ & $1,11^{\mathrm{f}}$ & $1,08-1,14$ \\
\hline
\end{tabular}

IC95\%: intervalo de confianza al 95\%; ITS: infección de transmisión sexual; RPc: razón de prevalencias crudas; RPa: razón de prevalencias ajustadas; *Porcentaje representa la prevalencia de la práctica en cada categoría de conocimiento; **Prueba de $\chi^{2} ;{ }^{* * *}$ Ajustado por ajustado por estado civil, región natural de vivienda, tipo de residencia, nivel de educación, nivel de pobreza y edad; *valor $p<0,05$; ${ }^{\text {valor }} p<0,001$. 


\section{Discusión}

Los resultados del presente estudio, con una muestra compleja de representatividad nacional, muestran que la mayoría de las MER peruanas respondieron correctamente a preguntas con respecto a la prevención y transmisión vía sexual del VIH. Además, se observa una asociación entre un conocimiento general adecuado sobre VIH con haber usado preservativo en la última relación sexual, con haber tenido una ITS en el último año y con haberse realizado alguna vez una prueba diagnóstica para la infección por VIH.

A pesar de un buen conocimiento sobre la transmisión sexual del VIH, se observa un deficiente conocimiento de las MER peruanas sobre la transmisión durante la gestación, parto y lactancia. Esto es menor en comparación estudios previos en MER de Etiopía y Bangladesh, donde a pesar de tener un conocimiento inadecuado general sobre la transmisión del VIH, tenían mejores conocimientos específicos sobre la transmisión durante el embarazo, parto o lactancia ${ }^{29,30}$. Sin embargo, los conocimientos sobre la transmisión durante el embarazo y lactancia en las MER peruanas son superiores en comparación con mujeres embarazadas de Pakistán y Etiopía $^{31,32}$. En general, las MER peruanas tienen mejor conocimiento sobre la transmisión materno-perinatal del VIH en comparación con mujeres embarazadas, pero menor en comparación con otras poblaciones de mujeres. Al 2017, el Perú se encuentra en proceso de alcanzar la eliminación de la transmisión materno-infantil del $\mathrm{VIH}^{33}$. Considerando que el presente estudio se realizó en mujeres no embarazadas, y que el embarazo no deseado es alto en mujeres peruanas, particularmente en adolescentes ${ }^{34,35}$ se requiere que se haga énfasis en las políticas públicas vinculadas a la salud sexual de las MER, priorizando, además del uso de métodos anticonceptivos, también los temas relacionados con la transmisión de VIH durante la gestación, parto y lactancia, enfocadas en un diagnóstico $\mathrm{y}$ tratamiento temprano ${ }^{36}$.

Un cuarto de las MER peruanas tenía un conocimiento adecuado sobre la infección por VIH. Esto es similar a lo que se reportó en dos estudios realizado en MER en Etiopía y Ghana, donde $25,2^{37}$ y $20,8 \% \%^{38}$ de la muestra estudiada tenían un conocimiento adecuado sobre VIH/ SIDA, respectivamente. A diferencia del presente estudio, éstos evaluaron el conocimiento adecuado con cinco preguntas claves sobre la transmisión sexual (uso de condón y pareja única) y conceptos erróneos sobre la trasmisión del virus (picadura de mosquito, compartir utensilios de comida, persona saludable con la infección por VIH) ${ }^{39}$. En general, las preguntas con mejor tasa de respuesta correcta fueron sobre la transmisión y prevención sexual del VIH, y algunas sobre conceptos erróneos, a excepción de la picadura de mosquitos. Esta relación entre las preguntas bien contestadas podría explicar la similitud de los resultados de los conocimientos adecuados sobre VIH/SIDA a pesar de usar formas de medición distintas. A diferencia de un estudio peruano que analizó las ediciones del ENDES 2004 a 2007 y encontró que 48\% (IC95\%: 46-49\%) de las mujeres encuestadas presentó un nivel de conocimiento adecuado sobre las ITS y VIH/SIDA. Esto fue porque definió el nivel de conocimiento a partir de la mediana de puntaje total de un grupo de preguntas ${ }^{24}$. La medición y comparación del nivel de conocimientos de VIH/SIDA depende del instrumento de medición para definir la variable. En este caso, el uso del tercil superior del puntaje total de 18 preguntas da resultados similares al del método de medición seguido por otros estudios en África utilizando cinco preguntas.

Una de cada cuatro MER peruanas y una de cada tres MER peruanas no casadas ni convivientes ha usado preservativo en la última relación sexual. Este resultado, en todas las mujeres, varía entre diferentes estudios. Es mayor en comparación con un estudio realizado en mujeres sexualmente activas en una región del norte del Perú ${ }^{40}$, es similar en mujeres de 15 a 44 años de Estados Unidos de América (E.U.A. $)^{41}$, pero menor a un estudio realizado en mujeres de 16 a 55 años de Sudáfrica ${ }^{42}$ y a un estudio realizado en mujeres de 18 a 44 años con comportamiento sexual de riesgo en E.U.A. ${ }^{43}$. El uso de preservativos en una relación sexual en las mujeres peruanas es bajo.

Esto podría estar condicionado por la capacidad de negociación de su uso de las $\mathrm{MER}^{44}$, especialmente en un contexto donde hay violencia por parte de la pareja ${ }^{45}$. Es necesario estudiar las razones por las que las MER no usan preservativos, especialmente aquellas que se encuentran en un contexto de violencia de género o que tienen relaciones sexuales con una pareja no estable.

Esto es similar a un estudio realizado en mujeres sexualmente activas en Brasil, el cual determinó que el conocimiento sobre el uso específico de preservativos se asocia con su uso adecuado ${ }^{46}$. En el presente estudio, la evaluación de los conocimientos sobre la infección por VIH y su trasmisión incluyen conocimiento sobre el uso de preservativo, cómo conseguirlo y su función. Entonces, probablemente los resultados obtenidos sean explicados por los conocimientos específicos sobre el uso de preservativo. Sin embargo, a pesar de este resultado, 82,6 y $78,2 \%$ de las encuestadas conoce sobre la efectividad y dónde conseguir un preservativo, respectivamente. Esto indica que aún es necesario mejorar el conocimiento específico sobre la prevención de la transmisión sexual del VIH.

El tener un conocimiento adecuado sobre la infección por VIH se asoció con haberse realizado una prueba diagnóstica para VIH y con haber tenido el diagnóstico de una ITS en el último año. Esto es similar a un estudio 
realizado en migrantes africanos en Alemania, el cual encontró que a mayor conocimiento sobre la infección por VIH aumenta la probabilidad de haberse realizado una prueba diagnóstica para la infección por $\mathrm{VIH}^{47}$. De manera retrospectiva, esta asociación tiene sentido, pues es probable que al momento de haberse realizado la prueba diagnóstica de VIH, la MER peruana haya recibido también información sobre la infección por VIH, aumentando así su nivel de conocimiento ${ }^{48}$. Por otro lado, nuestro resultado es diferente a un estudio realizado en jóvenes de Rusia que atienden a centros de salud, donde no se encontró asociación entre conocimientos sobre transmisión del VIH y ser diagnosticado con una ITS en ese momento ${ }^{49}$. Sin embargo, la posible explicación para esta asociación es que el hecho de haber tenido una ITS en el pasado, haya condicionado haber recibido un tratamiento médico y haber recibido consejería profesional. Esto, finalmente aumentaría los niveles de conocimientos en las MER que hubieran tenido una ITS en el último año.

El presente estudio tuvo la limitación del sesgo de información, ya que, no se pudo contar con mayor cantidad de variables importantes para esta relación, como lo son el haber tenido una pareja sexual ocasional, consumo de otras drogas durante el acto sexual, haber tenido relaciones sexuales con una persona que hubiera tenido múltiples parejas sexuales y el haber tenido relaciones sexuales vía oral y/o anal ${ }^{18,50}$. Adicionalmente, la medición de prácticas fue hecho por auto-reporte, por lo que, las respuestas de la encuesta pudieron estar condicionadas por diversos factores como: memoria, pudor y creencias religiosas, entre otras. Sin embargo, estudios previos utilizan el autoreporte como la forma para evaluar las prácticas ${ }^{17,31,32}$. A pesar de ello, el presente estudio tiene la fortaleza que, debido al tamaño muestral y estrategia de muestreo paramétrico de la base de datos primaria, los resultados podrían tener una aproximación nacional. Esto podría servir a las instituciones estatales y otras relacionadas, en propuestas de políticas educativas, de promoción y prevención de la infección por VIH enfocadas en generar más conocimiento y competencias, con el objetivo de cambiar prácticas.

Se concluye que, la mayoría de las MER peruanas tienen un adecuado nivel de conocimientos sobre la transmisión y prevención sexual de la infección por VIH, pero un bajo nivel de conocimientos en otros tipos de transmisión del VIH. Además, tienen una baja prevalencia de uso de preservativo en su última relación sexual. A pesar de ello, las MER con adecuado conocimiento general tienen mayor prevalencia de haber usado preservativo en su última relación sexual.

Agradecimientos: Al doctorando Jorge Maguiña, por el apoyo en el análisis estadístico para el uso del SVY.

\section{Referencias bibliográficas}

1.- Global HIV \& AIDS statistics - 2019 fact sheet [Internet]. [citado 18 de marzo de 2020]. Disponible en: https://www.unaids.org/en/ resources/fact-sheet

2.- UNAIDS. Key population [Internet]. Disponible en: https://www.unaids.org/en/topic/ key-populations [citado el 23 de julio de 2020].

3.- Gutiérrez B, Regla M. Implicaciones éticas, legales y sociales del diagnóstico de VIH/ sida en la mujer. Rev Cuba Salud Pública. 2013;39(1):124-34. https://www.scielosp.org/ pdf/rcsp/2013.v39n1/124-134/es

4.- Birdthistle I, Tanton C, Tomita A, de Graaf K, Schaffnit SB, Tanser F, et al. Recent levels and trends in HIV incidence rates among adolescent girls and young women in ten high-prevalence African countries: a systematic review and meta-analysis. Lancet Glob Health. 2019; 7(11):e1521-40. doi: 10.1016/S2214109X(19)30410-3.

5.- Facts and figures: HIV and AIDS [Internet]. UN Women. Disponible en: https://www.unwomen. org/en/what-we-do/hiv-and-aids/facts-andfigures [citado el 18 de marzo de 2020].

6.- Leddy A M, Weiss E, Yam E, Pulerwitz J.
Gender-based violence and engagement in biomedical HIV prevention, care and treatment: a scoping review. BMC Public Health. 2019;19: 897. doi: 10.1186/s12889-019-7192-4.

7.- Morgan R, Ayiasi RM, Barman D, Buzuzi S, Ssemugabo C, Ezumah N, et al. Gendered health systems: evidence from low- and middleincome countries. Health Res Policy Syst. 2018;16: 58. doi: s12961-018-0338-5.

8.- Closson K, McLinden T, Parry R, Lee M, Gibbs A, Kibel M, et al. Severe intimate partner violence is associated with all-cause mortality among women living with HIV. AIDS Lond Engl. 2020; 34(10): 1549-58. doi: 10.1097/ QAD.0000000000002581.

9.- Tsapalas D, Parker M, Ferrer L, Bernales M. Gender-based violence, perspectives in Latin America and the Caribbean. Hisp Health Care Int Off J Natl Assoc Hisp Nurses. 2020; 1540415320924768. doi: $10.1177 / 1540415320924768$

10.- The multiple faces of the intersections between HIV and violence against women [Internet] Development Connections, UNIFEM, Pan American Health Organization, Inter-American Commission of Women and the Latin American and Caribbean Women's Health Network;
2008. Disponible en: http://www.paho.org/hq/ dmdocuments/2009/HIV_VAW_EN.pdf [citado el 24 de julio de 2016].

11.- Benavides M, León J, Etesse M, Espezúa L, Stuart J. Exploring the association between segregation and physical intimate partner violence in Lima, Peru: The mediating role of gender norms and social capital. SSM - Popul Health. 2018; 7: 100338. doi: 10.1016/j. ssmph.2018.100338.

12.- Centro Nacional de Epidemiología, Prevención y Control de, Enfermedades. Situación epidemiológica del VIH-Sida en el Perú [Internet]. 2018. Disponible en: https:// www.dge.gob.pe/portal/docs/vigilancia/vih/ Boletin_2018/diciembre.pdf

13.- OMS | VIH/SIDA [Internet]. WHO. [citado 18 de marzo de 2020]. Disponible en: http://www. who.int/topics/hiv_aids/es/

14.- Rodríguez E C, Moreno R del C C, Naranjo H $\mathrm{S}$, García Raga M. Intervención educativa sobre VIH/SIDA en la ESBU: Guillermo González Polanco. Guisa, enero-abril 2010. Multimed. 2014;18(2):1-23. https://www.medigraphic. com/pdfs/multimed/mul-2014/mul142p.pdf.

15.- Lugo N S, Rivero L A A. VIH/SIDA: prevención y mercadeo social. Isla de la 
Juventud. Cuba. Horiz Sanit. 2 de agosto de 2014; 13 (2): 177-87. https://www.redalyc.org/ pdf/4578/457845287002.pdf.

16.- Harrison A, Colvin C J, Kuo C, Swartz A, Lurie M. Sustained high HIV incidence in young women in Southern Africa: social, behavioral, and structural factors and emerging intervention approaches. Curr HIV/AIDS Rep. 2015; 12(2): 207-15. doi: 10.1007/s11904-015-0261-0.

17.- Pei R, Ji-Ke C, Yu G, Yang Y, Nan L, Liao Q, et al. Sexual behaviors related to HIV infection in Yi women of childbearing age in rural areas of southwest China. AIDS Care. 2020; 1-5. doi: 10.1080/09540121.2020.1784445.

18.- Villegas N, Cianelli R, Santisteban D, Lara L, Vargas J. Factores que influencian la adquisición de infecciones de transmisión sexual y VIH en mujeres jóvenes chilenas que participaron en la intervención online I-STIPI. Hisp Health Care Int Off J Natl Assoc Hisp Nurses. 2016; 14(1): 47-56. doi: $10.1177 \% 2 \mathrm{~F} 1540415316629682$.

19.- Kenyon C R, Tsoumanis A, Schwartz I S. HIV prevalence correlates with high-risk sexual behavior in Ethiopia's regions. PloS One. 2015; 10 (10): e0140835. doi: 10.1371/journal. pone. 0140835 .

20.- Wand H, Reddy T, Ramjee G. Temporal trends in sexual behaviours and their impacts on HIV incidence among South African women: 2002-2016. AIDS Care. 2020; 1-7. doi: 10.1080/09540121.2020.1789054.

21.- El SIDA en cifras [Internet]. Programa Conjunto de las Naciones Unidas sobre el VIH/Sida; Disponible en: http://www.unaids. org/sites/default/files/media_asset/AIDS_by_ the_numbers_2015_es.pdf [citado el 23 de diciembre de 2019].

22.- Zetola N M, Modongo C, Olabiyi B, Ramogola-Masire D, Collman R G, Chao L-W. Examining the relationship between alcohol use and high-risk sex practices in a population of women with high HIV incidence despite high levels of HIV-related knowledge. Sex Transm Infect. 2014; 90(3): 216-22. doi: 10.1136/ sextrans-2013-051244.

23.- Patrício A C F de A, Bezerra V P, Nogueira J A, Moreira M A S P, Camargo B V, Santos J de S. Knowledge of sex workers about HIV/ AIDS and its influence on sexual practices. Rev Bras Enferm. 2019; 72(5): 1311-7. doi: 10.1590/0034-7167-2018-0590.

24.- Pernaz-Linsuy G, Cárcamo-Cavagnaro C. Conocimientos, actitudes y prácticas de la mujer peruana sobre la infección por VIH/ SIDA. Rev Peru Med Exp Salud Publica. 2015; 32(4): 667-72. doi: 10.17843/ rpmesp.2015.324.1756.

25.- Encuentro sobre prevención y otros servicios de ITS y VIH dirigidos a pueblos originarios indígenas amazónicos de Bolivia y Perú [Internet]. 2013. Disponible en: http://bvs.
minsa.gob.pe:81/local/MINSA/3019.pdf [citado el 24 de octubre de 2019].

26.- Estrategia Comunitaria Nuevo Sapasoa: Cuidado de la salud sexual y reproductiva y prevención del VIH en comunidades indígenas, a través de la estrategia de pares comunitarios [Internet]. Disponible en: http://bvs.minsa.gob. pe:81/local/MINSA/2200.pdf [citado el 24 de julio de 2019].

27.- Instituto Nacional de Estadística e Informática. Ficha Técnica. Encuesta Demográfica y de Salud Familiar 2019. Disponible en: https://proyectos.inei.gob.pe/endes/2019/ documentos_2019/FICHA_TECNICA_ ENDES\%202019.pdf.

28.- Hernández-Vásquez A, Chacón-Torrico H. Manipulación, análisis y visualización de datos de la Encuesta Demográfica y de Salud Familiar con el programa R. Rev Peru Med Exp Salud Pública. 2019; 36 (1): 128-33. doi: 10.17843/rpmesp.2019.361.4062.

29.- Yaya S, Bishwajit G, Danhoundo G, Shah V, Ekholuenetale M. Trends and determinants of HIV/AIDS knowledge among women in Bangladesh. BMC Public Health. 2016; 16(1): 812. doi: 10.1186/s12889-016-3512-0.

30.- Luba T R, Feng Z, Gebremedhin S A, Erena A N, Nasser AM, Bishwajit G, et al. Knowledge about mother-to-child transmission of HIV, its prevention and associated factors among Ethiopian women. J Glob Health. 2020; 7(2): 46-50. doi: 10.7189/jogh.07.020414.

31.- Irfan A, Kazmi S K, Anwar Z, Khan F M A, Khan J, Arif Y, et al. Knowledge and attitude of pregnant women regarding HIV transmission, prevention and associated factors in Karachi, Pakistan-A cross-sectional study. Sex Reprod Healthc. 2019; 21: 46-50. doi: 10.1016/j. srhc.2019.06.001.

32.- Abtew S, Awoke W, Asrat A. Knowledge of pregnant women on mother-to-child transmission of HIV, its prevention, and associated factors in Assosa town, Northwest Ethiopia. HIV/AIDS Auck1 NZ. 2016; 8: 101-7. doi: 10.2147/HIV.S100301.

33.- Organización Panamericana de la Salud. Nuevas generaciones sin la infección por el VIH, la sífilis, la hepatitis B y la enfermedad de Chagas en las Américas 2018 [Internet]. 2019. Disponible en: https://iris.paho.org/bitstream/ handle/10665.2/50993/9789275320679_spa. pdf? sequence $=1$ \&isAllowed $=y$

34.- Mendoza W, Subiría G. El embarazo adolescente en el Perú: situación actual e implicancias para las políticas públicas. Rev Peru Med Exp Salud Pública. 2013;30(3): 4719. doi: doi: 10.17843/rpmesp.2013.303.286.

35.- Instituto Nacional de Estadística e Informática. Perú. Encuesta Demográfica y de Salud Familiar 2019. Disponible en: https://www.inei. gob.pe/media/MenuRecursivo/publicaciones_ digitales/Est/Endes2019/.
36.- Baker A N, Bayer A M, Viani R M, Kolevic L, Sim M-S, Deville J G. Morbidity and mortality of a cohort of Peruvian HIV-infected children 2003-2012. Pediatr Infect Dis J. 2018; 37(6): 564-9. doi: 10.1097/INF.0000000000001865.

37.- Agegnehu C D, Geremew B M, Sisay M M, Muchie K F, Engida Z T, Gudayu T W, et al Determinants of comprehensive knowledge of HIV/AIDS among reproductive age (15-49 years) women in Ethiopia: further analysis of 2016 Ethiopian demographic and health survey. AIDS Res Ther. 2020; 17: 51. doi: 10.1186/ s12981-020-00305-z.

38.- Darteh E K M. Individual and contextual predictors of comprehensive HIV and AIDS knowledge among young females in Ghana. Afr J AIDS Res AJAR. 2020; 1-9. http://uaps2019. popconf.org/uploads/190696.

39.- Ochako R, Ulwodi D, Njagi P, Kimetu S, Onyango A. Trends and determinants of comprehensive HIV and AIDS knowledge among urban young women in Kenya. AIDS Res Ther. 2011; 8: 11. doi: 10.1186/1742-64058-11.

40.- Burgos-Muñoz S J, Toro-Huamanchumo C J. Zika knowledge and preventive practices among reproductive-age women from Lambayeque, Peru. Eur J Obstet Gynecol Reprod Biol. 2018; 228: 255-60. doi: 10.1016/j. ejogrb.2018.07.017.

41.- Copen C E. Condom use during sexual intercourse among women and men aged 1544 in the United States: 2011-2015 National Survey of Family Growth. Natl Health Stat Rep. 2017; 105: 1-18. PMID: 29155683.

42.- Ntshiqa T, Musekiwa A, Mlotshwa M, Mangold K, Reddy C, Williams S. Predictors of male condom use among sexually active heterosexual young women in South Africa, 2012. BMC Public Health. 2018; 18(1): 1137. doi: 10.1186/s12889-018-6039-8.

43.- Nasrullah M, Oraka E, Chavez P R, Johnson C $\mathrm{H}$, DiNenno E. Factors associated with condom use among sexually active US adults, National Survey of Family Growth, 2006-2010 and 2011-2013. J Sex Med. 2017; 14(4): 541-50. doi: 10.1016/j.jsxm.2017.02.015.

44.- Nesoff E D, Dunkle K, Lang D. The impact of condom use negotiation self-efficacy and partnership patterns on consistent condom use among college-educated women. Health Educ Behav. 2016; 43(1): 61-7. doi: 10.1177/1090198115596168.

45.- Peasant C, Sullivan T P, Ritchwood T D, Parra G R, Weiss N H, Meyer J P, et al. Words can hurt: the effects of physical and psychological partner violence on condom negotiation and condom use among young women. Women Health. 2018; 58(5): 483-97. doi: 10.1080/03630242.2017.1316345.

46.- Andrade S S da C, Zaccara A A L, Leite K N S, Brito K K G de, Soares M J G O, 


\section{VIH / SIDA}

doi: 10.1371/journal.pone.0194244.

Costa M M L, et al. Knowledge, attitude and practice of condom use by women of an impoverished urban area. Rev Esc Enferm USP. 2015; 49(3): 364-71. doi: 10.1590/S0080623420150000300002.

47.- Kuehne A, Koschollek C, Santos-Hövener C, Thorlie A, Müllerschön J, Mputu Tshibadi C, et al. Impact of HIV knowledge and stigma on the uptake of HIV testing - Results from a community-based participatory research survey among migrants from sub-Saharan Africa in Germany. PloS One. 2018; 13(4): e0194244.
48.- Paschen-Wolff M M, Reddy V, Matebeni Z, Southey-Swartz I, Sandfort T. HIV and sexually transmitted infection knowledge among women who have sex with women in four Southern African countries. Cult Health Sex. 2020; 22(6): 705-21. doi: 10.1080/13691058.2019.1629627.

49.- Shipitsyna E, Krasnoselskikh T, Zolotoverkhaya E, Savicheva A, Krotin P, Domeika M, et al. Sexual behaviours, knowledge and attitudes regarding safe sex, and prevalence of non-viral sexually transmitted infections among attendees of youth clinics in St. Petersburg, Russia: STI prevalence and predictors among young people in Russia. J Eur Acad Dermatol Venereol. 2013; 27(1): e75-84. doi: 10.1111/j.1468-3083.2012.04512.x

50.- Mmbaga E J, Leyna G H, Mnyika K S, Klepp K-I. Sexually transmitted infections knowledge and its impact in the practice of risky sexual behaviours and HIV serostatus: results from rural Kilimanjaro, Tanzania. Sex Transm Infect. 2008; 84(3): 224-6. doi: 10.1136/ sti.2007.029488. 